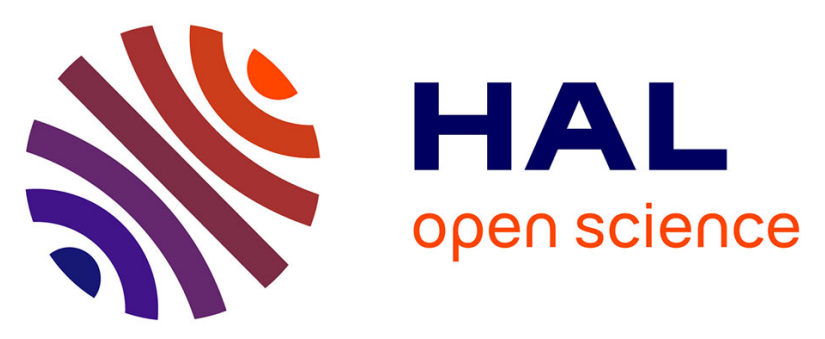

\title{
Two-Dimensional Growth of CdSe Nanocrystals, from Nanoplatelets to Nanosheets
}

\author{
Cécile Bouet, Benoit Mahler, Brice Nadal, Benjamin Abecassis, Mickael
} Tessier, Sandrine Ithurria, Xiangzhen Xu, Benoit Dubertret

\section{To cite this version:}

Cécile Bouet, Benoit Mahler, Brice Nadal, Benjamin Abecassis, Mickael Tessier, et al.. TwoDimensional Growth of CdSe Nanocrystals, from Nanoplatelets to Nanosheets. Chemistry of Materials, 2013, 25 (4), pp.639-645. 10.1021/cm304080q . hal-02995312

\section{HAL Id: hal-02995312 \\ https://hal.science/hal-02995312}

Submitted on 9 Nov 2020

HAL is a multi-disciplinary open access archive for the deposit and dissemination of scientific research documents, whether they are published or not. The documents may come from teaching and research institutions in France or abroad, or from public or private research centers.
L'archive ouverte pluridisciplinaire HAL, est destinée au dépôt et à la diffusion de documents scientifiques de niveau recherche, publiés ou non, émanant des établissements d'enseignement et de recherche français ou étrangers, des laboratoires publics ou privés. 


\section{D growth of CdSe nanocrystals, from nanoplatelets}

\section{to nanosheets.}

Cécile Bouet, Benoit Mahler, Brice Nadal, Benjamin Abecassis, Mickael D. Tessier, Sandrine Ithurria, Xiangzhen Xu, Benoit Dubertret*

ESPCI Paristech, 10 rue Vauquelin, 75005 Paris, France

Laboratoire de Physique des Solides, Univ. Paris-Sud, CNRS, UMR 8502, 91405 Orsay, France

KEYWORDS ()

ABSTRACT We report the continuous lateral extension of cadmium selenide nanoplatelets into nanosheets using continuous injection of precursors at high temperature. We show that we can obtain CdSe nanosheets with lateral dimensions up to $700 \mathrm{~nm}$ and a well defined thickness that can be tuned with atomic precision. When the nanosheet lateral size increases, they roll on themselves to form nano-scrolls that can unroll upon surface modification. The final geometry of the nanosheets can be tuned to different morphologies using precursors that favor the growth of specific crystal faces. We provide a detailed study of the CdSe nanosheets growth and their optimization for three different thicknesses. 
Introduction

Two dimensional semiconductor nanocrystals are structures with one dimension -their thickness- smaller than their lateral dimensions. These 2D structures can be produced using different techniques that include molecular beam epitaxy ${ }^{1}$, exfoliation ${ }^{2}$, and synthesis in solution $^{3-5}$. The latter method gives access to colloidal 2D structures, and has several advantages. It is rapid, gives access to a large quantity of materials with a control of their thickness ${ }^{6}$ that $^{\text {can }}$ be performed in some cases with atomic precision ${ }^{7}$. The objects obtained are easily processable through simple precipitation and resuspension in various solvents, and their surface chemistry can be changed at will using techniques similar to that developed for the spherical $\mathrm{NCs}^{8-10}$. This processability is of technological interest for the development of miniaturized electronic ${ }^{11,12}$ and optoelectronic devices ${ }^{13}$. One of the important features for their efficient use in device fabrication is the possibility to obtain colloidal 2D structures with tuneable, and preferably large lateral dimensions. Such objects could be an interesting alternative to their spherical ${ }^{14}$ and rodshaped $^{15}$ colloidal counter part, since their two-dimensional geometries are directly compatible with established device designs and processing approaches developed in the semiconductor industry. 2D semiconductor colloidal structures of various lateral dimensions and different compounds have recently been synthesized ${ }^{16-19}$. Amongst these materials, the cadmium based nanoplatelets have seen a rapid development because of their very interesting optical properties, including high fluorescence quantum yield ${ }^{5}$, fluorescence emission full width half maximum smaller than $12 \mathrm{~nm}^{4,5}$, and ultra-fast fluorescence lifetimes ${ }^{7}$. On the structural level it was also shown that the chalcogenide NPLs can be atomically flat ${ }^{5}$, and can synthesized with different crystal structures. The team of $\mathrm{T}$. Hyeon has reported the synthesis of wurtzite $\mathrm{CdSe}$ 
naonribbons ${ }^{4}$. This synthesis was extended to CdSe nanosheets ${ }^{20}$, doped CdSe nanoribbons ${ }^{21}$ and CdS nanoplates ${ }^{22}$. The team of Buhro extended the synthesis of Peng ${ }^{23}$ to synthesize wurtzite CdSe quantum belts ${ }^{24}$ that have morphological and crystalline properties very similar to the nanoribbons. Both team have studied the formation mechanisms of the wurtzite structures ${ }^{20,25}$. We, as well as the team of Peng ${ }^{26,27}$ have synthesized zinc blende NPLs. We have synthesized zinc blende CdSe NPLs with three different thicknesses that have precisely defined emission maxima at $462 \mathrm{~nm}, 513 \mathrm{~nm}$, and $553 \mathrm{~nm}$, when their lateral dimensions are larger than the 2D CdSe Bohr radius ${ }^{5}$. These populations with different thicknesses will be referred to in the remaining of the text as the 462 NPLs, the 513 NPLs and the 553 NPLs populations. Here we report a method for the continuous lateral extension of zinc blende CdSe nanoplatelets with lateral dimensions that can be as small as few nanometers into CdSe nanosheets with lateral dimensions that can be as large as $700 \mathrm{~nm}$. This method can be implemented either on existing nanoplatelets to extend them after their synthesis or, to continuously extend small clusters into nanoplatelets and then into nanosheets. We show that the lateral extension of the 2D nanoplatelets can be performed along different crystallographic directions depending on the precursors used during the reaction. The shape of the nanosheets, for example rectangular or polygonal, can be controlled to a certain extent.

\section{Experimental methods}

Chemicals: Cadmium acetate dihydrate $\left(\mathrm{Cd}(\mathrm{OAc})_{2} \cdot 2 \mathrm{H}_{2} \mathrm{O}\right)$, trioctylphosphine (TOP), technical grade 1-octadecene (ODE), myristic acid sodium salt, oleic acid (OA), butanol and selenium in powder were purchased from Sigma-Aldrich. Ethanol and hexane were purchased from SDS Carlo-Erba. 


\section{Precursor preparation:}

The preparation of cadmium myristate is performed as reported elsewhere. ${ }^{28}$

Preparation of TOP-Se at 2.24 M. In a glovebox, $1.77 \mathrm{~g}(22.4 \mathrm{mmol})$ of selenium in powder is suspended in $10 \mathrm{~mL}$ TOP, and stirred until complete dissolution. The solution is filtered using a PTFE $0.2 \mu \mathrm{m}$ filter.

Preparation of Se-ODE at 0.1 M. $140 \mathrm{~mL}$ of octadecene is degazed in a three-neck flask. Under Argon flow, the temperature is set to $180^{\circ} \mathrm{C}$. A solution of $1.18 \mathrm{~g}$ of selenium dispersed in $10 \mathrm{~mL}$ of octadecene is slowly introduced into the hot octadecene until complete dissolution, while the temperature is increased from $180^{\circ} \mathrm{C}$ to $205^{\circ} \mathrm{C}$. The solution is heated for 30 minutes at $205^{\circ} \mathrm{C}$.

Preparation of the injection solution. $0.36 \mathrm{mmol}$ of $\mathrm{Cd}(\mathrm{OAc})_{2} \cdot 2 \mathrm{H}_{2} \mathrm{O}$ are dissolved into $1 \mathrm{~mL}$ ethanol by mild heating. $0.12 \mathrm{mmol}$ of oleic acid and $0.43 \mathrm{mmol}$ of Se-ODE $0.1 \mathrm{M}$ are then added to the solution. $1 \mathrm{~mL}$ of butanol is added in order to have a monophasic solution. When TOP-Se is used as a selenium source in place of the Se-ODE, it is diluted in ODE and injected from another syringe in order to prevent the reaction from starting at room temperature in the injection syringe.

Synthesis and growth of extended nanosheets emitting at $462 \mathbf{n m} .15 \mathrm{~mL}$ of ODE are degassed for 15 minutes in a three-neck flask. The precursors solution is injected at $240{ }^{\circ} \mathrm{C}$ under Argon from one or two syringes, depending on the selenium precursor used, at a rate of $5 \mathrm{~mL} / \mathrm{h}$. A continuous argon flow through one septum of the three neck flask punctured with a needle 
helps the evaporation of the butanol and the ethanol during the reaction. At the end of the injection, $2 \mathrm{~mL}$ of oleic acid are added in the flask, which is then cooled down rapidly. The solution is diluted in hexane and centrifugated at $5000 \mathrm{rpm}$. The precipitate contains the $462 \mathrm{~nm}$ emitting nanosheets.

Synthesis of nanosheets emitting at $513 \mathbf{~ n m}$. Cadmium behenate is synthesized in situ via heating $0.35 \mathrm{mmol}$ of $\mathrm{Cd}(\mathrm{OAc})_{2} .2 \mathrm{H}_{2} \mathrm{O}$ with $0.7 \mathrm{mmol}$ of behenic acid in $\mathrm{ODE}$ under vacuum for 30 minutes in a three-neck flask. The temperature is decreased to room temperature and $0.3 \mathrm{mmol}$ of selenium powder dispersed in ODE are introduced in the mixture under Argon flow. The temperature is set to $240{ }^{\circ} \mathrm{C}$. As the temperature rises, $0.35 \mathrm{mmol}$ of cadmium acetate are introduced in the flask when the solution turns orange. After heating at $240{ }^{\circ} \mathrm{C}$ for 8 minutes, $2 \mathrm{~mL}$ of oleic acid are added and the solution is rapidly cooled down at room temperature. Hexane and ethanol are added to the reacting solution and centrifugated. The platelets precipitate while the quantum dots remain in the supernatant. After this step, if the solution contains 462 NSs in addition to $513 \mathrm{NSs}$, an additional centrifugation in hexane is performed and the precipitate, that contains a vast majority of $462 \mathrm{NSs}$, is discarded.

Synthesis of nanosheets emitting at $553 \mathbf{~ n m}$. The synthesis of $553 \mathrm{NCs}$ is similar to the one reported elsewhere ${ }^{29}$. In brief, $170 \mathrm{mg}$ of $\mathrm{Cd}(\mathrm{myr})_{2}$ and $14 \mathrm{~mL}$ of ODE are degazed in a threeneck flask. Under argon flow, the mixture is heated up to $250^{\circ} \mathrm{C} .12 \mathrm{mg}(0.15 \mathrm{mmol})$ of $\mathrm{Se}$ dispersed in $1 \mathrm{~mL}$ of ODE are injected followed one minute later by $120 \mathrm{mg}$ of $\mathrm{Cd}(\mathrm{OAc})_{2} \cdot 2 \mathrm{H}_{2} \mathrm{O}$ (0.45 mmol). The solution is heated for 15 minutes at $250{ }^{\circ} \mathrm{C}$.

Small Angle X-ray Scattering. SAXS experiments were performed at the SWING beamline of the SOLEIL synchrotron (Saint-Aubin, France). Measurements were carried out using a fixed 
energy of $12 \mathrm{keV}$ and three sample to detector positions $(0.85,1.07$ and $6.56 \mathrm{~m})$. The typical accessible range of scattering vector modulus $\mathrm{q}$ was $0.02-10 \mathrm{~nm}^{-1}(\mathrm{q}=4 \pi \sin \theta / \lambda$, where $2 \theta$ is the scattering angle and $\lambda=1.033 \AA$ the wavelength). Scattering patterns were recorded on an AVIEX 170170 CCD camera formed by four detectors and radially averaged.

\section{Results and Discussion}

We have first studied the lateral extension of the thinnest CdSe NPLs population with an emission maximum at $462 \mathrm{~nm}$, the 462 NPLs (Figure 1). It is based on a slow injection of the precursors solution of cadmium and of selenium in a solution of octadecene (ODE) heated at 240 ${ }^{\circ} \mathrm{C}$. Figure 1a shows the evolution versus time of the absorption spectra of a typical synthesis that lasted for 40 minutes. 30 seconds after the onset of the precursors' injection, two absorption peaks are already distinguishable with maximum respectively at $462 \mathrm{~nm}$ and around $437 \mathrm{~nm}$. These two peaks correspond respectively to the electron-heavy hole and electron-light hole transitions of the 462 NPLs, as previously reported ${ }^{5}$. This suggests that nanoplatelets have already formed after 30 seconds of injection. Transmission Electron Microscope (TEM) images of the reaction product after 30 seconds confirms the presence of nanoplatelets that have lateral dimensions close to $20 \mathrm{~nm}$ (Fig. 1b). The nanoplatelets are folded and stacked together, and some nanoplatelets appear clearly on their side. No other smaller nanoparticles such as the small clusters forming the nanoplatelets precursors are observed ${ }^{30}$. These observations suggest that the supersaturation threshold of monomers concentration above which the nucleation of nanoparticles starts is quickly reached just at the beginning of the injection, leading to the nucleation of small nanoplatelets. After this initial nucleation stage, the concentration of monomers decreases and the additional precursors introduced during the continuous injection react with the nanoplatelets already formed to extend them laterally. 10 minutes after the onset of 
the injection, the lateral extension of the nanoplatelets is visible on the TEM images. Note that the absorption spectra are crude extracts of the reaction media diluted in hexane. After 40 minutes of reaction, the absorption spectrum has not evolved much, apart from a small absorption local maximum at around $512 \mathrm{~nm}$, corresponding to the one-monolayer thicker 513 NPLs population. We confirmed the continuous lateral extension of the NPLs after the first 30s with TEM images (Fig. 1b). After 10 minutes, we observe that the CdSe nanoplatelets roll up on themselves to form nanoscrolls. Such rolling of thin nanomembrane of nanosheet has already been reported in the case of exfoliated, or strained semiconductor structures ${ }^{31}$, but to the best of our knowledge it is the first time that such regular, paper like folding, is reported for colloidal 2D structures. The length of the nanoscrolls depends on the reaction time; from $130 \mathrm{~nm}$ on average after 10 minutes reaction, it grows to $300 \mathrm{~nm}$ after 40 minutes reaction. The diameter of the nanoscrolls does not change during the reaction and is close to $30 \mathrm{~nm}$. When observed with High Resolution Transmission Electron Microscopy (HRTEM), the walls of the nanoscrolls appear as alternating bright/dark regions with regular spacing and that are extended almost continuously along the scroll length. This suggests that the nanoscrolls are formed of one nanosheet that is rolled on itself instead of several nanosheets aggregated together.

Figure 2 shows Small-Angle X-rays Scattering (SAXS) pattern of dispersion of NSs in hexane after the synthesis. This technique is well suited to obtain structural information on nanomaterials in solutions on scales ranging from one to hundreds of nanometers. The scattered intensity comes from the contrast of electronic density between the electron-rich CdSe and the electron-poor solvent. In the small wave vector region $\left(\mathrm{q}<10^{-2} \mathrm{~nm}^{-1}\right)$, we observe marked oscillations of the scattered intensity at $\mathrm{q}=0.134,0.324$ and $0.524 \mathrm{~nm}^{-1}$. This wave vector range corresponding to spatial dimensions between 10 to $50 \mathrm{~nm}$, we attribute these oscillations to the 
radial dimension of the nanoscrolls. Getting a more precise value of the tube diameter would necessitate the full fitting of the scattering profile to a theoretical model, which is out of the scope of this manuscript. However, since the scattering signal is an ensemble average of a large collection of nanoparticles in solution, the amplitude of the oscillations indicates that the distribution of diameter of the tubes is monodisperse with a small deviation around the average.

At higher $\mathrm{q}$, a series of three Bragg peaks are observed at $\mathrm{nq}^{*}$ with $\mathrm{q}^{*}=1.19 \mathrm{~nm}^{-1}$ and $\mathrm{n}$ ranging from 1 to 3 . These peaks and their indexation are consistent with a lamellar order within the shell of the nanoscrolls caused by the folding of the nanoplatelets on themselves. From $\mathrm{q}^{*}$, the distance between the stacks can be estimated to $\mathrm{d}^{*}=2 \pi / \mathrm{q}^{*}=5.3 \mathrm{~nm}$. With a platelet thickness of $0.9 \mathrm{~nm}$ we deduce that the distance between two platelets edges is $4.4 \mathrm{~nm}$. This yields an extension of 2.2 $\mathrm{nm}$ per capping ligand (oleic acid). to be compared with a maximum extension of an 18 carbons chain $\left(2.47 \mathrm{~nm}\right.$ according to the Tanford formula $\left.{ }^{32}\right)$ showing a small overlapping of the two facing hydrocarbon chains. Altogether, the SAXS experiment confirms the structure observed by TEM and yield a detailed quantitative information on the stacking of the platelets. Furthermore, these experiments were realized on liquid dispersions and confirm that the folding of the nanosheets happens in the solution and not on the TEM grid. The formation of the nanoscrolls points to an asymmetric source of strain in the nanoplatelets. One asymmetry we could find in these zinc blende structures is the arrangement of the cadmium atoms that pave the two largest facets of the nanosheets (see Fig. S1). The alignment of the cadmium atoms from the top and the bottom plane are rotated by 90 degrees with respect to each other, and the carboxylate ligands that are bound to these cadmium atoms may introduce an asymmetric strain on the nanoplatelets that could explain the formation of the nanoscrolls. More detailed studies and modelisation are necessary to understand in details this unusual folding. Although the lengthening of the 
nanoscrolls suggests that the nanoplatelets continue their extension during the reaction, we had no information on the actual lateral dimensions or the shape of the nanosheets we had synthesized. We investigated several methods to unfold the nanoscrolls, and we found out that the growth of one layer of CdS on each side of the CdSe nanoscroll induces their unfolding. TEM images of the unfolding nanosheets are represented below their rolled counterparts in Figure 1c. The unfolded nanosheets are almost squares, which suggests that the nanoplatelets grow laterally along two directions with similar kinetics. A SAXS pattern of unfolded nanoplatelets is shown on figure 2 . We observe that the oscillations of the scattered intensity at low q (figure 2.A) have disappeared to be replaced by a monotonous decay following a power law with exponent -2.6 . Hence, the scattering signature of the radius of the tube has disappeared. However, large platelets perfectly dispersed in solution should yield a scattered intensity decaying as $\mathrm{q}^{-2}$. This discrepancy indicates that the large unfolded platelets are aggregated in solution. Furthermore, the Bragg peaks at higher scattering vectors (figure 2.B), which were attributed to the stacking of the nanoplatelets within the tubular structure, have also disappeared and are replaced by two much broader features shifted towards smaller wave vectors $(0.8$ and 1.6 $\mathrm{nm}^{-1}$ ) also caused by a short term order in aggregates of thicker platelets. Altogether, these observations are consistent with the unfolding of the nanoscrolls into wide, flat plates, which are aggregated in solutions. The lateral dimensions of the nanosheets are typically $300 \mathrm{~nm}$, but they can reach up to $700 \mathrm{~nm}$ (see Figure S5). These wide dimensions can be achieved only with a careful choice of the synthesis parameters such as the temperature, the injection rate, the nature and the concentration of the ligands. We now discuss the influence of these parameters.

We first explored the influence of the temperature on the nanoplatelets extension, in the range $180{ }^{\circ} \mathrm{C}-260{ }^{\circ} \mathrm{C}$, keeping all the other parameters of the synthesis constant. For all the 
temperatures tested, the population emitting at $462 \mathrm{~nm}$ is always predominant, as seen on the absorption spectra (figure S2a). TEM images of the NPLs (figure S2b) synthesized and extended at different temperatures up to $240{ }^{\circ} \mathrm{C}$ show that the higher the temperature, the greater the lateral extension. At high temperature, the nucleation of the first nanoplatelets happens faster than at lower temperature, when fewer precursors have been injected in the solution. This results in a lower concentration of small, initial, nanoplatelets which can further extend laterally. For temperatures $>240^{\circ} \mathrm{C}$, aggregated quantum dots are concomitantly formed with the NPLs, as illustrated in figure S2e, and selective precipitation does not permit to separate them from the nanosheets. When the temperature is lower than $220^{\circ} \mathrm{C}$, we could not obtain nanoplatelets with dimensions larger than 100nm, suggesting the continuous nucleation of new nanoplatelets during the injection. We conclude that the optimal temperature to obtain the largest nanosheets while avoiding secondary nucleation is around $240^{\circ} \mathrm{C}$.

We have also investigated the influence of the injection rate when the temperature was fixed at $220^{\circ} \mathrm{C}$. We tested several injection rates, from $11.5 \mathrm{~mL} / \mathrm{h}$ to $69 \mathrm{~mL} / \mathrm{h}$. We found that when the precursors are added at too high a rate $(>69 \mathrm{~mL} / \mathrm{h})$, only small NPLs are obtained. Indeed, when the concentration of monomers in the solution exceeds at all time the supersaturation level at which nucleation starts ${ }^{33}$, small nanoplatelets nucleate continuously, their concentration is high and their lateral dimensions small. In the other hand, when the injection rate is low (figure S2c), the supersaturation level is never reached after the initial nucleation stage, and the precursors are consumed to grow existing NPLs only. However, if the injection is too low $(5.75 \mathrm{ml} / \mathrm{h})$, QDs as well as other NPLs populations (figure S3) start to appear, as it is the case when high temperature $\left(260^{\circ} \mathrm{C}\right)$ is used for the growth (figure $\left.\mathrm{S} 2 \mathrm{~b}\right)$. 
We also explored the influence of the length of the carboxylic acid ligand used during the lateral extension of the nanoplatelets. Whatever the carbon chain length of the carboxylic acid we tested, 462 NPLs only are formed with our injection protocol. For short carbon chains, such as octanoic or undecanoic acid, we obtained nanoplatelets with smaller lateral dimensions that tend to aggregate easily (Fig. S4), suggesting that long chain ligands favor the growth of the NPLs instead of their nucleation. We found that oleic acid is the most efficient ligand to obtain the largest NPLs.

We have seen that temperature, injection rate and size of the carboxylic chains have a strong influence on the final size and the purity of the nanosheets we synthesize. We now explore the influence of the precursor of selenium on the growth kinetics and the morphology control of the NPLs. Similar studies have been performed for CdSe nanocrystals ${ }^{34}$, using di-n-octyl phosphine and di-n-octylphosphinic acid. Here, we choose to compare the extension of NPLs performed with Se-ODE and with Se complexed with Tri-n-octyl phosphine in various stoichiometries. These two selenium precursors have been studied in details for the formation of CdSe nanoscrystals $^{35,36}$. The lateral extension is performed with stoichiometric TOP-Se $(2.24 \mathrm{M})$ with a protocol similar to the one optimized for Se-ODE: temperature of $240{ }^{\circ} \mathrm{C}$ and injection rate of $69 \mathrm{ml} / \mathrm{h}$. As for Se-ODE, the nanosheets extended with TOP-Se have a first absorbance peak at $462 \mathrm{~nm}$ (figure S6). The concentration of Se in the TOP solution is important, if an excess of TOP remains in solution (for example, when TOP-Se $1 \mathrm{M}$ is used), NPLs are synthesized along with spherical QDs. On the contrary, when stoichiometric TOP-Se (TOP-Se $2.4 \mathrm{M}$ ) the cosynthesis of QDs is eliminated. Figure 3 shows TEM images of three syntheses, performed either with Se-ODE (top), or TOP-Se (middle and bottom). The synthesis referred to as TOP-Se*4 (figure 3, bottom) was performed with an injection of 4 times more precursors than the other 
(figure 3, middle), but the injection rates were similar for the two syntheses. For all the conditions we tested, the NPLs fold to form nanoscrolls (figure 3, left column), but the folding direction of the nanoscrolls does not depend on the Se precursor used. The nanoscrolls made with Se-ODE fold with one of their long edge first, whereas in the ones made with TOP-Se fold with one of their tip first, as represented schematically in figure 3a. To investigate the origin of the folding difference between these NPLs, we unfolded the nanoscrolls using a CdS overgrowth as described previously, to obtain flat nanosheets (figure 3b). The unfolded nanosheets revealed different shapes: rectangles in the case of Se-ODE and TOP-Se*4, and polygons with a larger number of facets when a lower amount of TOP-Se is used. FFT performed on HRTEM images of unfolded nanosheets (Figure 3) helps to assign the crystalline orientations of the NPLs with respect to their shape. First, the 4-fold symmetry of the diffraction patterns confirms that the NPLs thickness is parallel to a major crystal axis that we choose as $[001]^{30,37}$. For Se-ODE, the edges are found to be parallel to the (110) and (1-10) plans (Figure 3, top). When TOP-Se is used as a selenium precursor, additional facets are present and are parallel to the (100) and (010) plans. In this type of syntheses, NPLs are formed with facets that end with cadmium atoms only (100) and (010) as well as facets that end with a mixture of selenium and cadmium atoms (110) and (1-10). When more precursors are injected, however, the facets parallel to the (110) and (110) directions disappear, and rectangular nanosheets are obtained with facets parallel to the major crystal axis only. We thus conclude that when TOP-Se is used, both facets parallel to the (110) and (100) plans grow together. They have comparable stability, but the (100) facets are slightly more stable in our growth conditions and they end up to be the only facets left when more precursors are injected. On the contrary, when Se-ODE is used, the NPLs facets are only parallel to the (110) (or (1-10)) direction and the most stable facets are those formed of a mixture 
of $\mathrm{Cd}$ and Se atoms (Figure 3). Interestingly, the folding direction of the nanoscrolls does not depend on the Se precursor used even if the lateral facets differ. They fold along the [110] direction whatever the exposed facets are. More experiments need to be carried out to confirm the reason for NPLs folding.

The continuous injection procedure described above leads to 462 NPLs only. We were not able to make thicker NPLs directly by slow injection of the precursors but we could inject the precursors solution on seeds of thicker NPLs. To extend thicker NPLs, we have used a seeded growth approach with a continuous injection of precursors in a solution of small pre-synthesized thicker NPLs. Seeded growth extension of NPLs has already been reported ${ }^{30}$, but was limited to small NPLs. In brief, after the initial synthesis of thicker NPLs, a selective precipitation is performed to isolate a thickness-pure population of NPLs that are used as seeds. The injection solution is similar to the ones used for the 462 NPLs, and the quantity of seeds introduced in the flask is computed such that the extended NPLs have a surface 15 to 20 times larger than the seeds (see Table S1). The absorption spectra before and after the continuous injection on 513 NPLs are represented in figure 5a along with the corresponding TEM images. During the lateral extension, the NPLs first exciton absorption peak slightly shifts (around $1 \mathrm{~nm}$ ) to the red. This red shift takes place at the very beginning of the extension (See figure S7 in the Supporting Information) and suggests that the NPLs lateral dimensions rapidly exceed the Bohr radius of 2D CdSe systems. Starting with a population of $31 \mathrm{~nm}$ x $9 \mathrm{~nm} 513 \mathrm{NPLs}$, we manage to extend them to a roughly homogeneous population with dimensions close to $70 \mathrm{~nm} \times 55 \mathrm{~nm}$, close to a 14 times increase of the NPLs initial dimensions. However, some precursors are lost into the thicker population of NPLs that are nucleated, as suggested by the absorption peak at $550 \mathrm{~nm}$, characteristic of 553 NPLs. It is interesting to notice that the same experimental conditions (same 
precursors quantities, same temperature: $240^{\circ} \mathrm{C}$, same injection speed: $69 \mathrm{~mL} / \mathrm{h}$ ) lead to very different results whether if seeds are present in the initial solution or not. If no seeds are present, only 462 NPLs are synthesized, as demonstrated above. If seeds of 513 NPLs are present in the solution, only 513 NPLs are obtained, with a limited secondary nucleation of thicker NPLs. This observation further confirms the NPLs lateral extension mechanism through continuous reaction of precursors that we first proposed recently ${ }^{30}$. The seeded lateral extension of 513 NPLs has been extended to a thicker population: 553 NPLs. In this case, small NPLs with an emission maximum at $550 \mathrm{~nm}$ were introduced in ODE prior to the precursor injection, and the temperature had to be increased up to $260^{\circ} \mathrm{C}$ to avoid nucleation of thinner NPLs. The absorption spectra before and after injection are shown in figure 5b. A red shift (around $4 \mathrm{~nm}$ ) is also observed during the lateral extension that is confirmed by TEM images: the NPLs extend from $30 \mathrm{~nm} \times 6 \mathrm{~nm}$ to NPLs that are $100 \mathrm{~nm} \times 25 \mathrm{~nm}$. The final NPLs are 15 times wider than the seeds. The emission spectra before and after the lateral extension are presented in figure 5 for the two thicknesses studied. After extension, the two NPLs populations loose most of their fluorescence, although the loss is stronger for the thinner NPLs. This loss of fluorescence upon lateral extension could be due to incomplete ligand passivation of the very large NPLs surfaces. Further investigation is needed to determine the reason of the quantum yield loss. In spite of their large lateral dimensions these thicker NSs do not fold into nanoscrolls. Thicker NPLs are more rigid and may be stiff enough to resist to the constrains imposed by the ligands on the NPL surface.

\section{Conclusion}

We have shown that we could synthesize CdSe NPLs with lateral dimension that could reach 700 $\mathrm{nm}$. During the extension, the NPLs original thickness remains unchanged, so that we obtain 
nanosheets with a thickness that is controlled with atomic precision. The lateral extension using continuous injection of precursors has been performed on NPLs of different thicknesses, so that we obtain CdSe nanosheets with different thickness that differ by just one CdSe monolayer. The thinnest CdSe nanosheet fold on themselves during the lateral extension to form nanoscrolls that unfold when an additional CdS layer is grown on the CdSe nanoscrolls. The folding of the CdSe nanosheet is not observed on thicker samples. We have hypothesized that the folding of the nanosheet results from the asymmetric constraints imposed by the carboxylic acid ligands on the large cadmium-rich NPL surface. As it is the case in the growth of CdSe quantum dots into nanorods, we show that different facets of the NPLs can grow at different speed that can be controlled with the ligands used during the synthesis. These data should help better understand the control of the shape of large spherical QDs. The lateral size that we obtain should make the use and the integration of NPLs in devices easier and could open the way to single NPLs device, if properly connected.

Supporting Information. TEM images and absorption spectra of nanosheets made at different temperatures, with different injection rates, different ligands. Evolution in time of the absorption of 513 and 553 NSs. TEM images and absorption spectra of CdS and CdTe nanosheets. This material is available free of charge via the Internet at http://pubs.acs.org.

\section{Corresponding Author}

*benoit.dubertret@espci.fr

\section{Author Contributions}

The manuscript was written through contributions of all authors. All authors have given approval to the final version of the manuscript. 


\section{ABBREVIATIONS}

NSs, nanosheets; NLPs, nanoplatelets

\section{REFERENCES}

(1) Weisbuch, C.; Vinter, B. Quantum Semiconductor Structures : fundamentals and applications; Academic Press, 1991.

(2) Coleman, J. N.; Lotya, M.; O'Neill, A.; Bergin, S. D.; King, P. J.; Khan, U.; Young, K.; Gaucher, A.; De, S.; Smith, R. J.; Shvets, I. V.; Arora, S. K.; Stanton, G.; Kim, H. Y.; Lee, K.; Kim, G. T.; Duesberg, G. S.; Hallam, T.; Boland, J. J.; Wang, J. J.; Donegan, J. F.; Grunlan, J. C.; Moriarty, G.; Shmeliov, A.; Nicholls, R. J.; Perkins, J. M.; Grieveson, E. M.; Theuwissen, K.; McComb, D. W.; Nellist, P. D.; Nicolosi, V. Science 2011, 331, 568.

(3) Sigman, M. B.; Ghezelbash, A.; Hanrath, T.; Saunders, A. E.; Lee, F.; Korgel, B. A. J Am Chem Soc 2003, 125, 16050.

(4) Joo, J.; Son, J. S.; Kwon, S. G.; Yu, J. H.; Hyeon, T. J. Am. Chem. Soc. 2006, $128,5632$.

(5) Ithurria, S.; Dubertret, B. J. Am. Chem. Soc. 2008, 130, 16504.

(6) Vaughn, D. D.; In, S. I.; Schaak, R. E. Acs Nano 2011, 5, 8852.

(7) Ithurria, S.; Tessier, M. D.; Mahler, B.; Lobo, R. P. S. M.; Dubertret, B.; Efros, A. L. Nature Materials 2011, 10, 936.

(8) Rosen, E. L.; Buonsanti, R.; Llordes, A.; Sawvel, A. M.; Milliron, D. J.; Helms, B. A. Angew Chem Int Edit 2012, 51, 684.

(9) Nag, A.; Kovalenko, M. V.; Lee, J. S.; Liu, W. Y.; Spokoyny, B.; Talapin, D. V. J Am Chem Soc 2011, 133, 10612.

2012.

(10) Mahler, B.; Nadal, B.; Bouet, C.; Patriarche, G.; Dubertret, B. J Am Chem Soc

(11) Ko, H.; Takei, K.; Kapadia, R.; Chuang, S.; Fang, H.; Leu, P. W.; Ganapathi, K.; Plis, E.; Kim, H. S.; Chen, S. Y.; Madsen, M.; Ford, A. C.; Chueh, Y. L.; Krishna, S.; Salahuddin, S.; Javey, A. Nature 2010, 468, 286.

(12) Radisavljevic, B.; Radenovic, A.; Brivio, J.; Giacometti, V.; Kis, A. Nat. Nanotechnol. 2011, 6, 147.

(13) Kwak, J.; Bae, W. K.; Lee, D.; Park, I.; Lim, J.; Park, M.; Cho, H.; Woo, H.; Yoon, D. Y.; Char, K.; Lee, S.; Lee, C. Nano Letters 2012, 12, 2362.

$466,474$.

(14) Dong, A. G.; Chen, J.; Vora, P. M.; Kikkawa, J. M.; Murray, C. B. Nature 2010,

(15) Baker, J. L.; Widmer-Cooper, A.; Toney, M. F.; Geissler, P. L.; Alivisatos, A. P. Nano Letters 2010, 10, 195.

(16) Vaughn, D. D.; Patel, R. J.; Hickner, M. A.; Schaak, R. E. J. Am. Chem. Soc. 2010, 132, 15170.

(17) Kirkeminde, A.; Ruzicka, B. A.; Wang, R.; Puna, S.; Zhao, H.; Ren, S. Q. ACS Appl. Mater. Interfaces 2012, 4, 1174.

(18) Park, K. H.; Jang, K.; Son, S. U. Angew Chem Int Ed Engl 2006, 45, 4608.

(19) Jang, J. T.; Jeong, S.; Seo, J. W.; Kim, M. C.; Sim, E.; Oh, Y.; Nam, S.; Park, B.; Cheon, J. J Am Chem Soc 2011, 133, 7636. 
(20) Son, J. S.; Wen, X. D.; Joo, J.; Chae, J.; Baek, S. I.; Park, K.; Kim, J. H.; An, K.; Yu, J. H.; Kwon, S. G.; Choi, S. H.; Wang, Z. W.; Kim, Y. W.; Kuk, Y.; Hoffmann, R.; Hyeon, T. Angew. Chem.-Int. Edit. 2009, 48, 6861.

(21) Yu, J. H.; Liu, X. Y.; Kweon, K. E.; Joo, J.; Park, J.; Ko, K. T.; Lee, D.; Shen, S. P.; Tivakornsasithorn, K.; Son, J. S.; Park, J. H.; Kim, Y. W.; Hwang, G. S.; Dobrowolska, M.; Furdyna, J. K.; Hyeon, T. Nature Materials 2010, 9, 47.

(22) Son, J. S.; Park, K.; Kwon, S. G.; Yang, J.; Choi, M. K.; Kim, J.; Yu, J. H.; Joo, J.; Hyeon, T. Small 2012, 8, 2394.

(23) Pradhan, N.; Goorskey, D.; Thessing, J.; Peng, X. G. J Am Chem Soc 2005, 127, 17586.

(24) Liu, Y. H.; Wayman, V. L.; Gibbons, P. C.; Loomis, R. A.; Buhro, W. E. Nano Lett 2010, 10, 352.

(25) Liu, Y. H.; Wang, F. D.; Wang, Y. Y.; Gibbons, P. C.; Buhro, W. E. J. Am. Chem. Soc. 2011, 133, 17005.

(26) Li, Z.; Peng, X. G. J Am Chem Soc 2011, 133, 6578.

(27) Li, Z.; Qin, H. Y.; Guzun, D.; Benamara, M.; Salamo, G.; Peng, X. G. Nano Res. 2012, 5, 337.

(28) Yang, Y. A.; Wu, H. M.; Williams, K. R.; Cao, Y. C. Angew. Chem.-Int. Edit. 2005, 44, 6712 .

(29) Ithurria, S.; Tessier, M. D.; Mahler, B.; Lobo, R. P. S. M.; Dubertret, B.; Efros, A. Nat Mater 2011, 10, 936.

(30) Ithurria, S.; Bousquet, G.; Dubertret, B. J. Am. Chem. Soc. 2011, 133, 3070.

(31) Huang, M. H.; Cavallo, F.; Liu, F.; Lagally, M. G. Nanoscale 2011, 3, 96.

(32) Tanford, C. J Phys Chem-Us 1972, 76, 3020.

(33) Park, J.; Joo, J.; Kwon, S. G.; Jang, Y.; Hyeon, T. Angew Chem Int Edit 2007, 46, 4630.

(34) Wang, F. D.; Buhro, W. E. J Am Chem Soc 2012, 134, 5369.

(35) Bullen, C.; van Embden, J.; Jasieniak, J.; Cosgriff, J. E.; Mulder, R. J.; Rizzardo, E.; Gu, M.; Raston, C. L. Chem Mater 2010, 22, 4135.

(36) Evans, C. M.; Evans, M. E.; Krauss, T. D. J. Am. Chem. Soc. 2010, 132, 10973.

(37) Peng, X. G.; Li, Z. J. Am. Chem. Soc. 2011, 133, 6578. 
a)

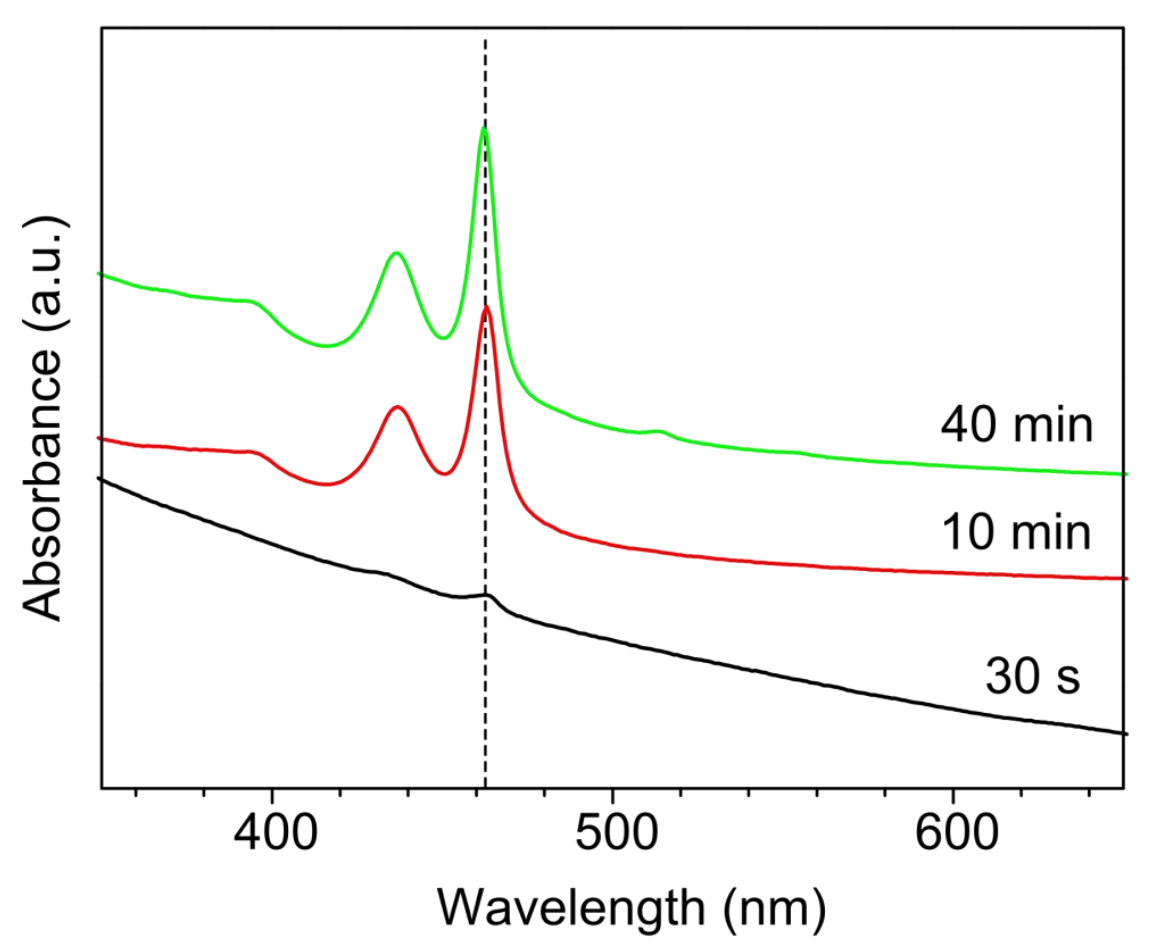

b)
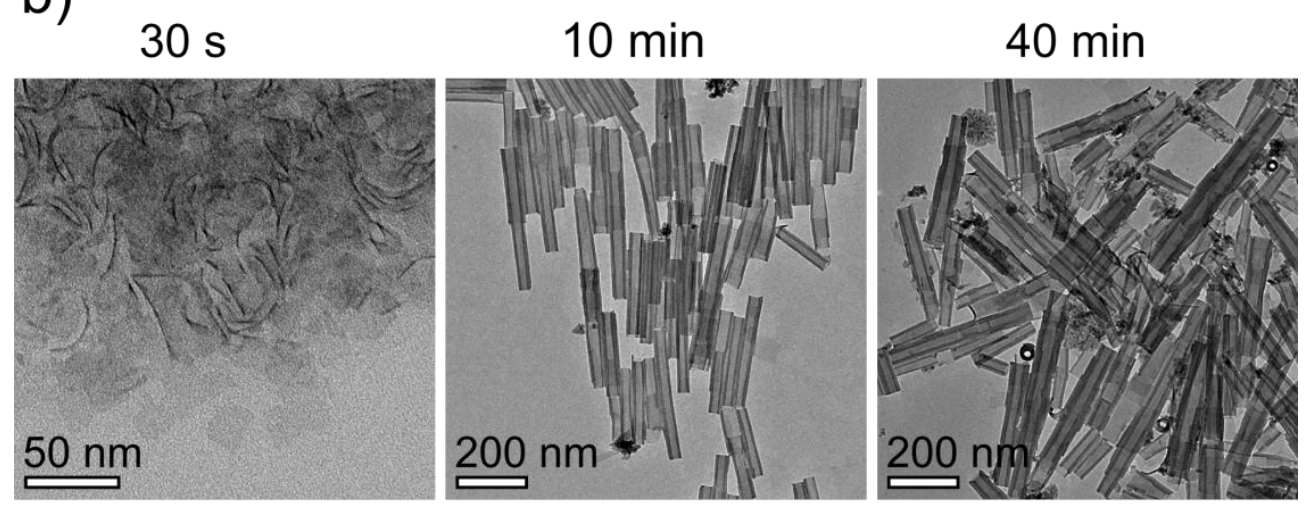

c)

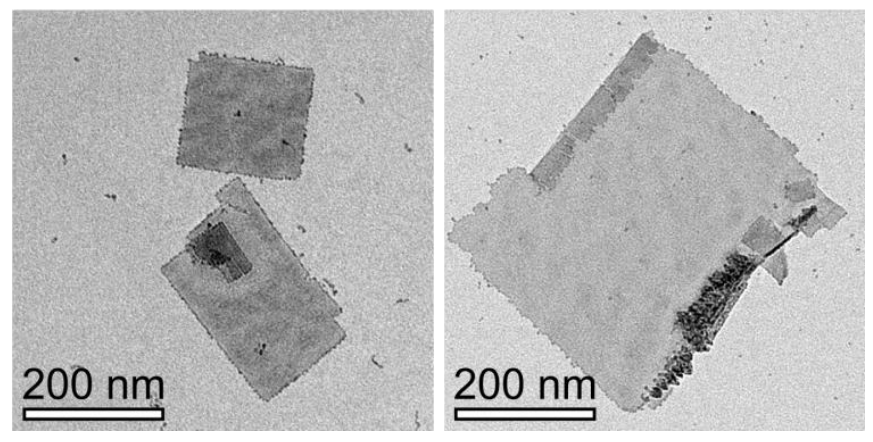

Figure 1.Nanosheets formation and continuous growth: a) Absorption spectra from aliquots taken at 30s, 10 min and 40 min; b) Corresponding TEM images showing $20 \mathrm{~nm}$ wide 
nanosheets after $30 \mathrm{~s}, 180 \mathrm{~nm}$ after $10 \mathrm{~min}$ and $300 \mathrm{~nm}$ after $40 \mathrm{~min}$; c) TEM images of the unfolded nanosheets after $10 \mathrm{~min}$ and $40 \mathrm{~min}$.

A)

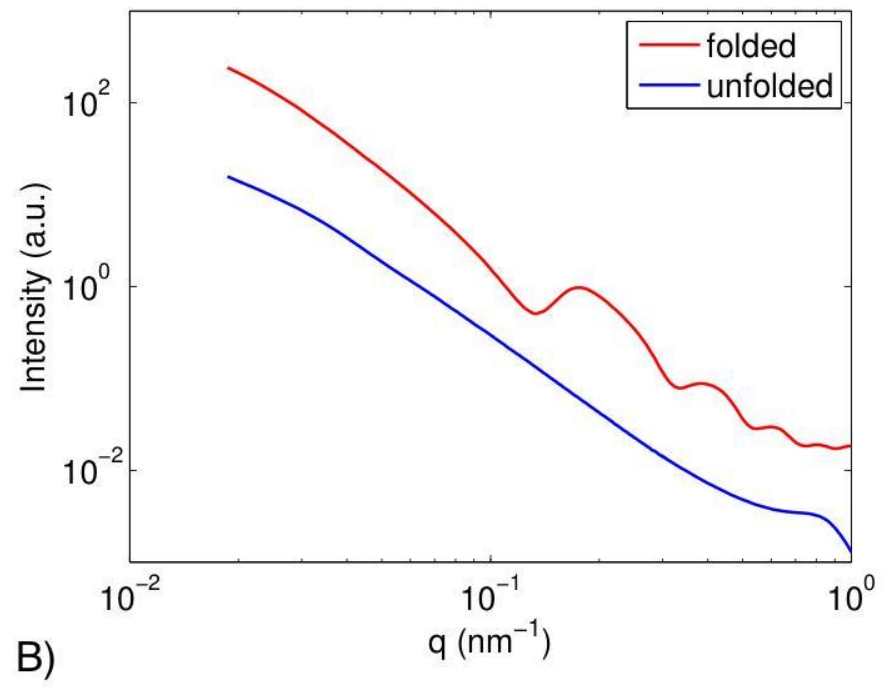

C)
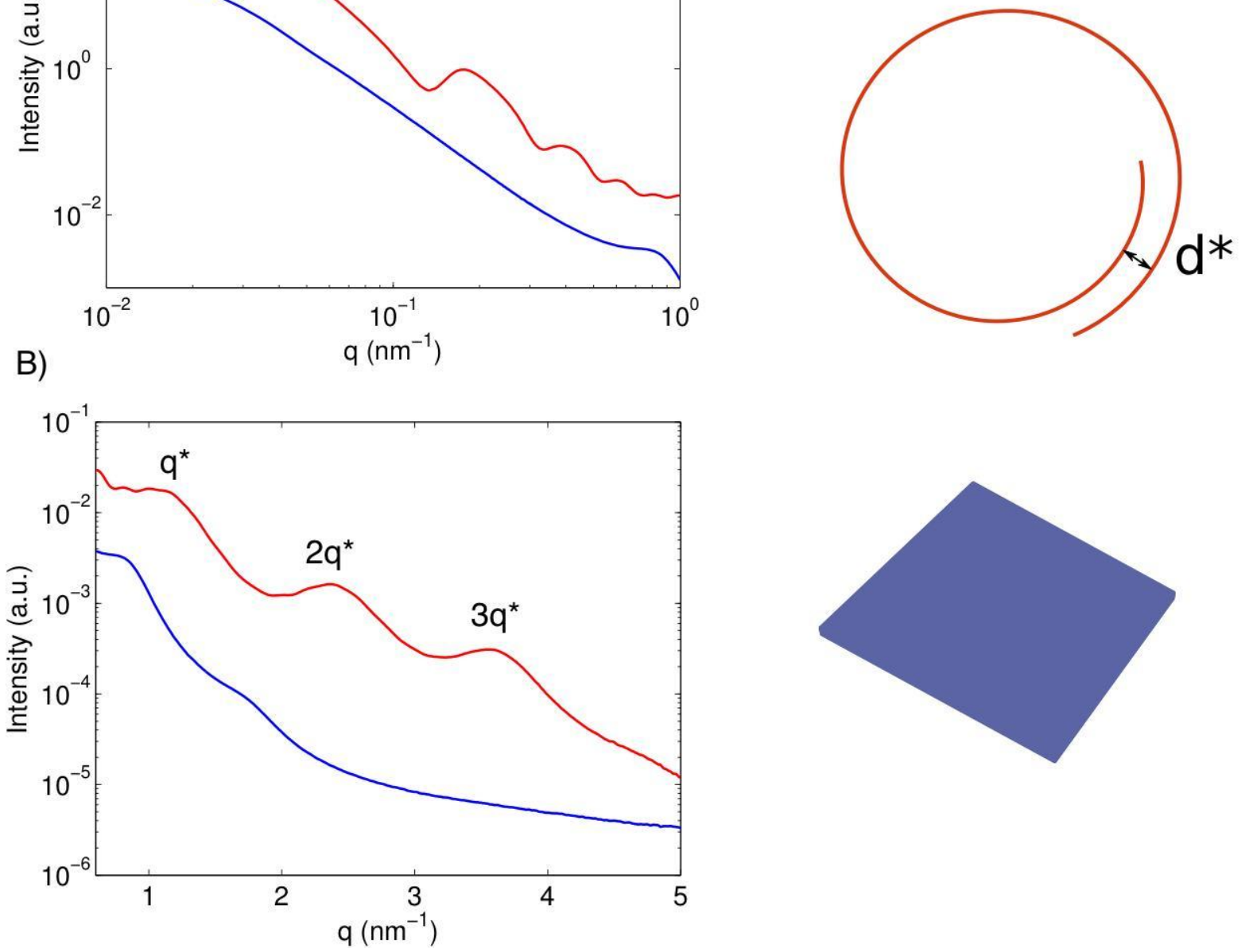

Figure 2. Small-Angle X-Ray Scattering: A) Low q scattering pattern for unfolded and folded nanoplatelets. B) High q scattering pattern for the same samples. C) Schematic representation of the structure of the nanoplatelets. The distance $\mathrm{d}^{*}$ corresponds to the broad Bragg peaks visible at high q values. 

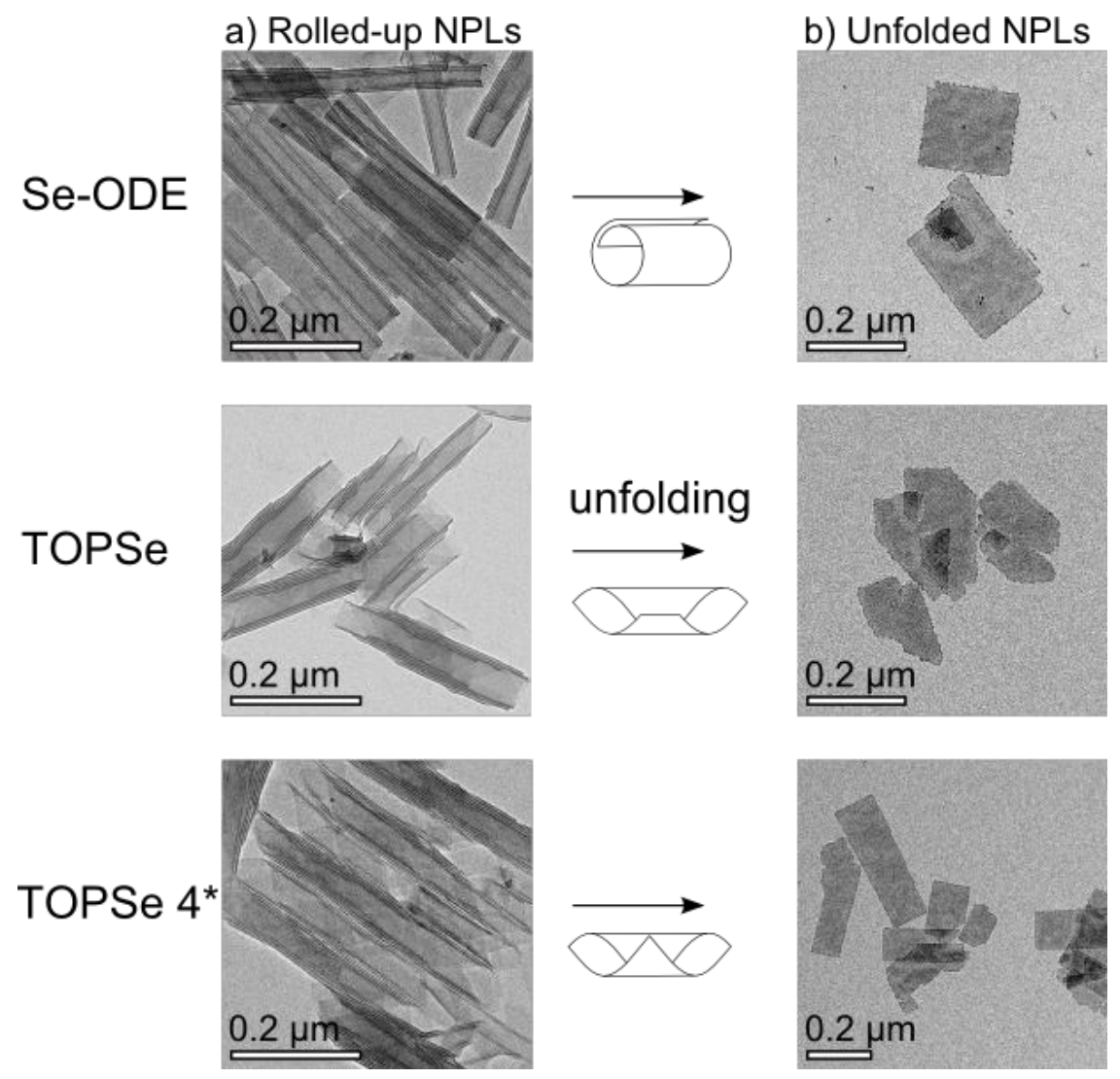

Figure 3 Facets control: from left to right, TEM images after the synthesis, and after unfolding. First row, nanosheets synthesized with Se-ODE. Second row, NSs synthesized using TOPSe. Third row, NSs synthesized using TOPSe. 
a) HRTEM

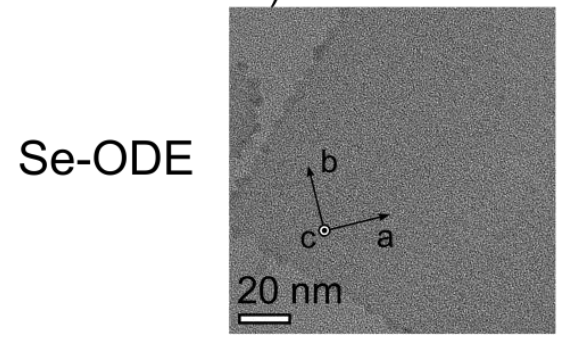

TOPSe
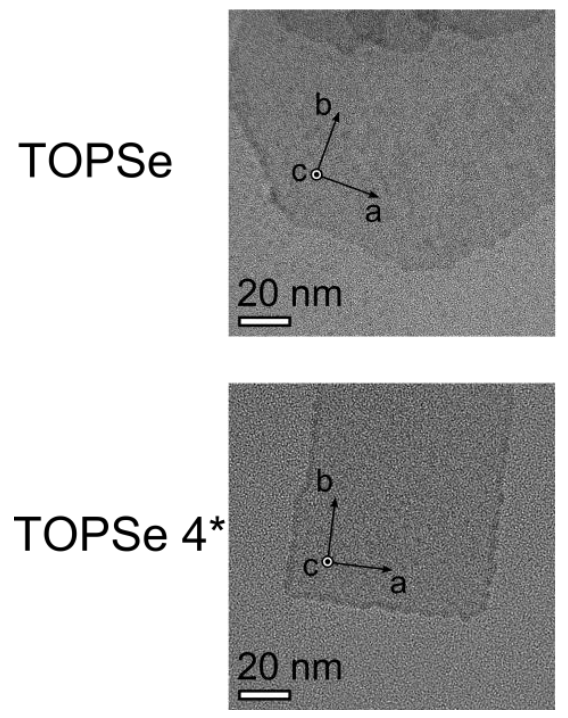

b) FFT
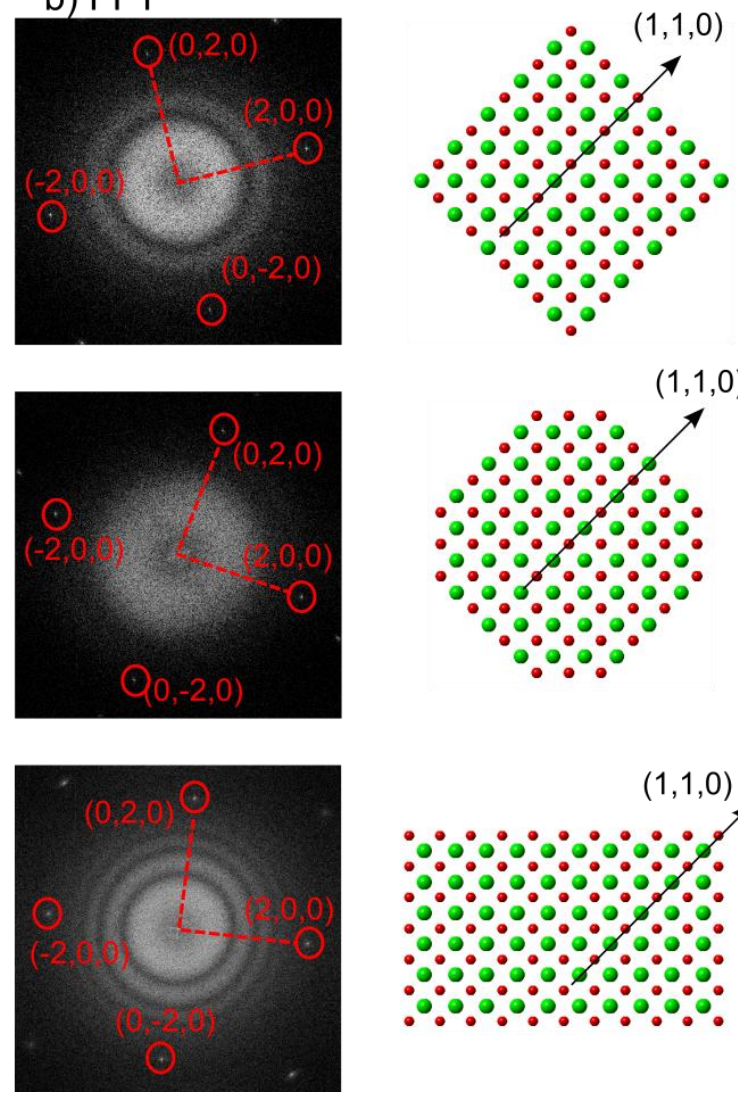

$(1,1,0)$

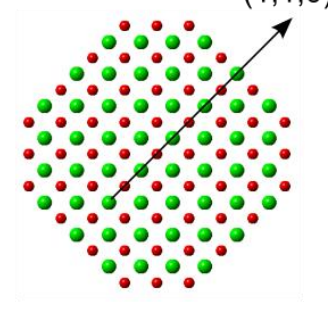

$(1,1,0)$

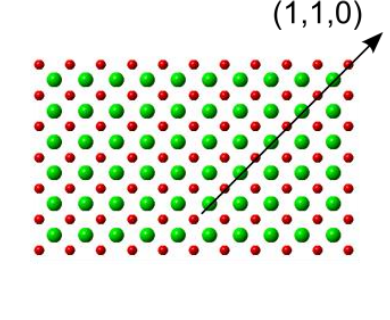

Figure 4. Facets control: from left to right, HRTEM images of the NPLs after unfolding and FFT on the HRTEM images. First row, nanosheets synthesized with Se-ODE, exhibiting only (110) facets. Second row, NSs synthesized using TOPSe exhibiting both (110) and (100) facets. Third row, NSs synthesized using TOPSe exhibiting only (100) facets. On the right, the corresponding model of the platelets with its folding axis has been represented. 
a)

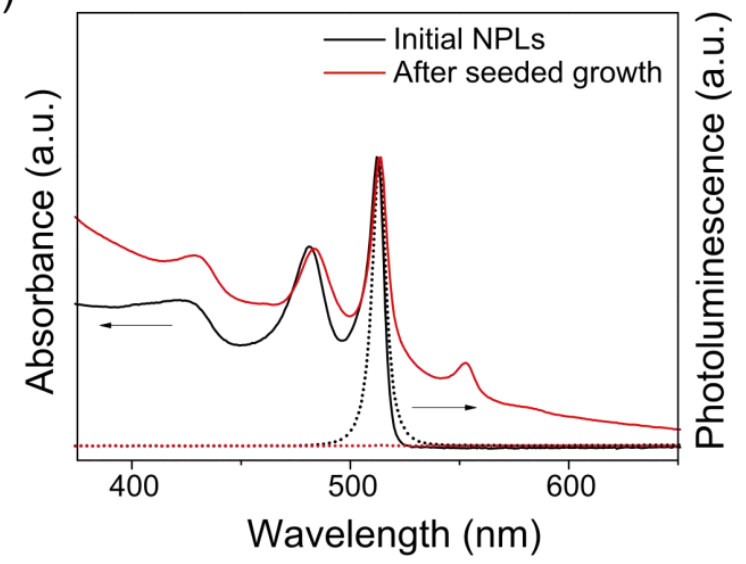

Initial NPLs

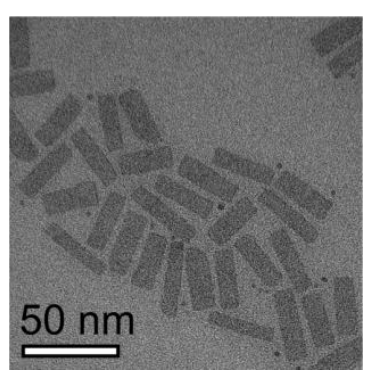

After growth

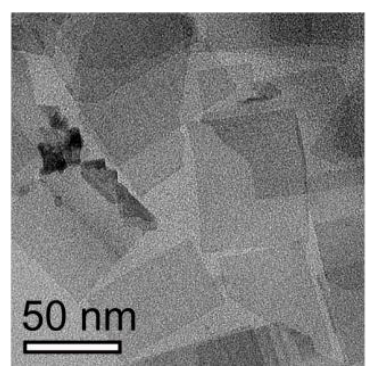

b)

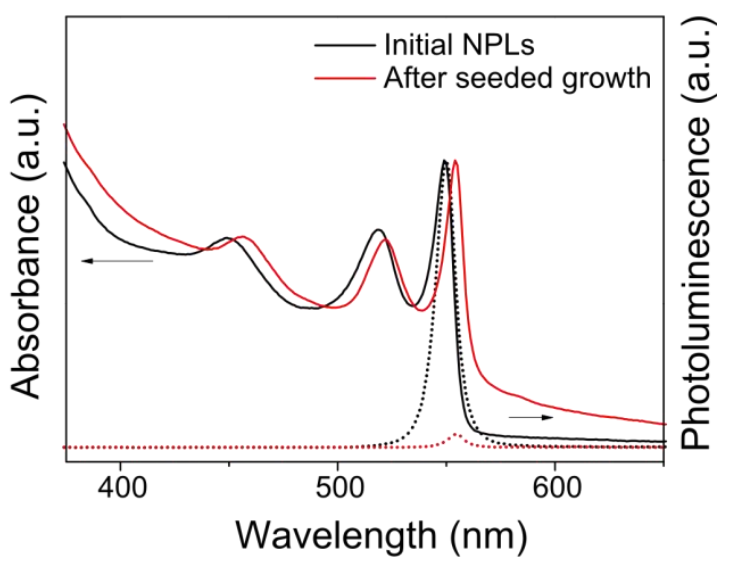

Initial NPLs

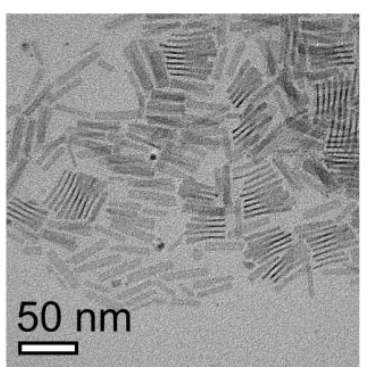

After growth

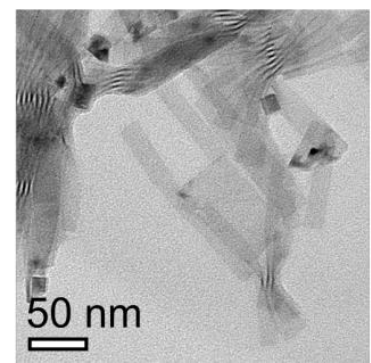

Figure 5. Lateral extension of a) 513 nanosheets and b) 553 nanosheets. Absorption and TEM images of the initial NPLs and the nanosheets after a continuous injection of precursors. 